\title{
ON THE PROJECTION CONSTANTS OF SOME TOPOLOGICAL SPACES AND SOME APPLICATIONS
}

\author{
ENTISARAT EL-SHOBAKY, SAHAR MOHAMMED ALI, \\ AND WATARU TAKAHASHI
}

Received 13 May 2001

We find a lower estimation for the projection constant of the projective tensor product $X \otimes^{\wedge} Y$ and the injective tensor product $X \otimes^{\vee} Y$, we apply this estimation on some previous results, and we also introduce a new concept of the projection constants of operators rather than that defined for Banach spaces.

\section{Introduction}

If $Y$ is a closed subspace of a Banach space $X$, then the relative projection constant of $Y$ in $X$ is defined by

$$
\lambda(Y, X):=\inf \{\|P\|: P \text { is a linear projection from } X \text { onto } Y\} .
$$

And the absolute projection constant of $Y$ is defined by

$$
\lambda(Y):=\sup \{\lambda(Y, X): X \text { contains } Y \text { as a closed subspace }\} .
$$

It is well known that any Banach space $Y$ can be isometrically embedded into $l_{\infty}(\Gamma)$ for some index set $\Gamma$ ( $\Gamma$ is usually taken to be $U_{Y^{*}}$ where $Y^{*}$ denotes the dual space of $Y$ and $U_{Y^{*}}$ denotes the set $\left.\left\{f: f \in Y^{*},\|f\| \leq 1\right\}\right)$ and that if $Y$ is complemented in $l_{\infty}(\Gamma)$, then it is complemented in every Banach space containing it as a closed subspace, that is, $Y$ is injective. We also know that for any such embedding the supremum in (1.2) is attained, that is, $\lambda(Y)=$ $\lambda\left(Y, l_{\infty}(\Gamma)\right)$ (see $\left.[1,4]\right)$. For each finite-dimensional space $Y_{n}$ with $\operatorname{dim} Y_{n}=n$, Kadets and Snobar [6] proved that $\lambda\left(Y_{n}\right) \leq \sqrt{n}$. König [7] showed that for each prime number $n$ the space $l_{n^{2}}^{\infty}$ contains an $n$-dimensional subspace $Y_{n}$ with projection constant

$$
\lambda\left(Y_{n}\right)=\sqrt{n}-\left(\frac{1}{\sqrt{n}}-\frac{1}{n}\right) .
$$

Copyright (C) 2001 Hindawi Publishing Corporation Abstract and Applied Analysis 6:5 (2001) 299-308 2000 Mathematics Subject Classification: 47B20, 46B10 URL: http://aaa.hindawi.com/volume-6/S1085337501000598.html 
König and Lewis [9] verified the strict inequality $\lambda\left(Y_{n}\right)<\sqrt{n}$ in case $n \geq 2$. Lewis [14] showed that

$$
\lambda\left(Y_{n}\right) \leq \sqrt{n}\left[1-n^{-2}\left(\frac{1}{5}\right)^{2 n+11}\right] .
$$

König and Tomczak-Jaegermann [11] also showed that there is a sequence $\left\{X_{n}\right\}_{n \in \mathbb{N}}$ of Banach spaces $X_{n}$ with $\operatorname{dim} X_{n}=n$ such that

$$
\lim _{n \rightarrow \infty} \frac{\lambda\left(X_{n}\right)}{\sqrt{n}}=1 .
$$

In fact, it is shown in [9] that for each Banach space $Y_{n}$ with dimension $n$, $\lambda\left(Y_{n}\right) \leq \sqrt{n}-c / \sqrt{n}$, where $c>0$ is a numerical constant and the $n$-dimensional spaces $X_{n}$ satisfy $\sqrt{n}-2 / \sqrt{n} \leq \lambda\left(X_{n}\right)$. The improvement of these results was given in [12], where an upper estimate for $\lambda\left(Y_{n}\right)$ was found in the form

$$
\lambda\left(Y_{n}\right) \leq \begin{cases}\sqrt{n}-\frac{1}{\sqrt{n}}+O\left(n^{-3 / 4}\right), & \text { in the real field, } \\ \sqrt{n}-\frac{1}{2 \sqrt{n}}+O\left(n^{-3 / 4}\right), & \text { in the complex field. }\end{cases}
$$

The precise values of $l_{n}^{1}, l_{n}^{2}$, and $l_{n}^{p}, 1<p<\infty, p \neq 2$, have been calculated by Grünbaum [4], Rutovitz [15], Gordon [3], and Garling and Gordon [2]. In the case of $1<p<2$, the improvement of these results was given by König, Schütt, and Tomczak-Jaegermann in [10], they showed that

$$
\lim _{n \rightarrow \infty} \frac{\lambda\left(l_{n}^{p}\right)}{\sqrt{n}}= \begin{cases}\sqrt{\frac{2}{\pi}}, & \text { in the real field, } \\ \frac{\sqrt{\pi}}{2}, & \text { in the complex field. }\end{cases}
$$

Some other results are mentioned in [2, 3, 13, 15].

For finite codimensional subspaces, Garling and Gordon [2] showed that if $Y$ is a finite codimensional subspace of the Banach space $X$ with codimension $n$, then for every $\epsilon>0$ there exists a projection $P$ from $X$ onto $Y$ with norm

$$
\|P\| \leq 1+(1+\epsilon) \sqrt{n} .
$$

\section{Notations and basic definitions}

The sets $X, Y, Z$, and $E$ denote Banach spaces, $X^{*}$ denotes the conjugate space of $X$ and $U_{X}$ denotes the unit ball of the space $X$. Elements of $X, Y, X^{*}$, and $Y^{*}$ will be denoted by $x, u, \ldots, y, v, \ldots, f, h, \ldots$, and $g, k, \ldots$, respectively. The 
injective tensor product $X \otimes^{\vee} Y$ between the normed spaces $X$ and $Y$ is defined as the completion of the smallest cross norm on the space $X \otimes Y$ and the norm on the space $X \otimes Y$ is defined by

$$
\left\|\sum_{i=1}^{n} x_{i} \otimes y_{i}\right\|_{X \otimes^{\vee} Y}=\sup \left|\sum_{i=1}^{n} f\left(x_{i}\right) g\left(y_{i}\right)\right|,
$$

where the supremum is taken over all functionals $f \in U_{X^{*}}$ and $g \in U_{Y^{*}}$.

The projective tensor product $X \otimes^{\wedge} Y$ between the normed spaces $X$ and $Y$ is defined as the completion of the largest cross norm on the space $X \otimes Y$ and the norm on $X \otimes Y$ is defined by

$$
\left\|\sum_{i=1}^{n} x_{i} \otimes y_{i}\right\|_{X \otimes^{\wedge} Y}=\inf \left\{\sum_{j=1}^{m}\left\|u_{j}\right\|\left\|v_{j}\right\|\right\},
$$

where the infimum is taken over all equivalent representations $\sum_{j=1}^{m} u_{j} \otimes v_{j} \in$ $X \otimes Y$ of $\sum_{i=1}^{n} x_{i} \otimes y_{i}$ (see [5]).

If $X$ is a Banach space on which every linear bounded operator from $X$ into any Banach space $Y$ is nuclear (this is the case in all finite-dimensional Banach spaces $X$ ), then for any Banach space $Y$ the space $X \otimes^{\vee} Y$ is isomorphically isometric to $X \otimes^{\wedge} Y$ (see [16]).

The set $\Omega=\left\{(f, g): f \in U_{X^{*}}, g \in U_{Y^{*}}\right\}=U_{X^{*}} \times U_{Y^{*}}$.

We start with the following two lemmas.

Lemma 2.1. For Banach spaces $X$ and $Y$ there is a norm one projection from $l_{\infty}\left(U_{X^{*}}\right) \otimes\left(\vee\right.$ or $\left.{ }^{\wedge}\right) l_{\infty}\left(U_{Y^{*}}\right)$ onto $l_{\infty}(\Omega)$.

Proof. Since the space $l_{\infty}(\Omega)$ has the 1-extension property, it is sufficient to show that $l_{\infty}(\Omega)$ can be isometrically embedded in the space $l_{\infty}\left(U_{X^{*}}\right) \otimes(\vee$ or $\wedge)$ $l_{\infty}\left(U_{Y^{*}}\right)$. In fact, every nonzero element $0 \neq \mathfrak{F}=\{\mathfrak{F}((f, g))\}_{f \in U_{X^{*}}, g \in U_{Y^{*}}}$ in the space $l_{\infty}(\Omega)$, (note that the norm in this Banach space is given by $\left.\|\mathfrak{F}\|_{l_{\infty}(\Omega)}=\sup _{f \in U_{X^{*}}} \sup _{g \in U_{Y^{*}}}|\mathfrak{F}((f, g))|\right)$ defines two scalar-valued functions $F \in l_{\infty}\left(U_{X^{*}}\right)$ and $G \in l_{\infty}\left(U_{Y^{*}}\right)$ by the following formulas:

$$
F(f)=\sup _{g \in U_{Y^{*}}}|\mathfrak{F}((f, g))|, \quad G(g)=\sup _{f \in U_{X^{*}}}|\mathfrak{F}((f, g))| .
$$

Clearly the element $\mathfrak{F}=\left(1 /\|\mathfrak{F}\|_{l_{\infty}(\Omega)}\right) \times(F \otimes G)$ is an element of the space $l_{\infty}\left(U_{X^{*}}\right) \otimes^{(\vee \text { or } \wedge)} l_{\infty}\left(U_{Y^{*}}\right)$. Since both the injective and the projective tensor products are cross norms, $\|\mathfrak{F}\|_{l_{\infty}\left(U_{\left.X^{*}\right) \otimes(\vee \text { or } \wedge)} l_{\infty}\left(U_{Y^{*}}\right)\right.}=\|\mathfrak{F}\|_{l_{\infty}(\Omega)}$. The mapping $J$ defined by the formula $J(\mathfrak{F})=\mathfrak{F}$ is the required isometric embedding.

Lemma 2.2. Let $X$ and $Y$ be two Banach spaces. Then $\lambda\left(X \otimes^{\vee} Y\right)=\lambda\left(X \otimes^{\vee} Y\right.$, $\left.l_{\infty}(\Omega)\right)$. 
302 On the projection constants of some topological spaces

Proof. It is also sufficient to show that the space $X \otimes^{\vee} Y$ can be isometrically embedded in $l_{\infty}(\Omega)$. In fact, every element $\mathfrak{F}=\sum_{i=1}^{n} x_{i} \otimes y_{i} \in X \otimes \vee Y$ defines a scalar-valued bounded function $\mathfrak{F} \in l_{\infty}(\Omega)$ by the formula $\mathfrak{F}((f, g))=$ $\sum_{i=1} f\left(x_{i}\right) g\left(y_{i}\right)$. Using definition (2.1) for the injective tensor product, we have $\|\mathfrak{F}\|_{\vee}=\|\mathfrak{F}\|_{l_{\infty}(\Omega)}$. The mapping $i$ defined by the formula $i(\mathfrak{F})=\mathfrak{F}$ is the required isometric embedding.

We have the following theorem.

THEOREM 2.3. (1) If $Y_{1}$ and $Y_{2}$ are complemented subspaces of Banach spaces $X_{1}$ and $X_{2}$, respectively, then the injective (resp., projective) tensor product $Y_{1} \otimes^{\vee} Y_{2}$ (resp., $Y_{1} \otimes^{\wedge} Y_{2}$ ) of the spaces $Y_{1}$ and $Y_{2}$ is complemented in the injective (resp., projective) tensor product $X_{1} \otimes^{\vee} X_{2}$ (resp., $X_{1} \otimes^{\wedge} X_{2}$ ) of the spaces $X_{1}$ and $X_{2}$ and

$$
\lambda\left(Y_{1} \otimes^{(\vee \text { or } \wedge)} Y_{2}, X_{1} \otimes^{(\vee \text { or } \wedge)} X_{2}\right) \leq \lambda\left(Y_{1}, X_{1}\right) \lambda\left(Y_{2}, X_{2}\right) .
$$

(2) If $X$ and $Y$ are injective spaces, then the space $X \otimes^{\vee} Y$ is injective. Moreover,

$$
\lambda\left(X \otimes^{\vee} Y\right) \leq \lambda(X) \lambda(Y) .
$$

Proof. Let $P_{1}$ and $P_{2}$ be any projections from $X_{1}$ onto $Y_{1}$ and from $X_{2}$ onto $Y_{2}$, respectively. Then the operator $P$ from the space $X_{1} \otimes^{\vee} X_{2}$ onto the space $Y_{1} \otimes^{\vee} Y_{2}$ (resp., from the space $X_{1} \otimes^{\wedge} X_{2}$ onto the space $Y_{1} \otimes^{\wedge} Y_{2}$ ) defined by

$$
P\left(\sum_{i=1}^{n} x_{i} \otimes y_{i}\right)=\sum_{i=1}^{n} P_{1}\left(x_{i}\right) \otimes P_{2}\left(y_{i}\right)
$$

is a projection and its norm $\|P\|$ is not exceeding $\left\|P_{1}\right\|\left\|P_{2}\right\|$. In fact, let $\sum_{i=1}^{n} x_{i} \otimes y_{i}$ be any element of the space $X_{1} \otimes^{(\vee \text { or } \wedge)} X_{2}$. Then, in the case of projective tensor product we have

$$
\begin{aligned}
\left\|P\left(\sum_{i=1}^{n} x_{i} \otimes y_{i}\right)\right\|_{Y_{1} \otimes \wedge Y_{2}} & =\left\|\sum_{i=1}^{n} P_{1}\left(x_{i}\right) \otimes P_{2}\left(y_{i}\right)\right\|_{Y_{1} \otimes \wedge Y_{2}} \\
& =\left\|\sum_{j=1}^{m} P_{1}\left(u_{i}\right) \otimes P_{2}\left(v_{i}\right)\right\| \|_{Y_{1} \otimes \wedge Y_{2}} \\
& \leq\left\|P_{1}\right\|\left\|P_{2}\right\| \sum_{j=1}^{m}\left\|u_{j}\right\|\left\|v_{j}\right\|,
\end{aligned}
$$


for all equivalent representations $\sum_{j=1}^{m} u_{j} \otimes v_{j}$ of $\sum_{i=1}^{n} x_{i} \otimes y_{i}$. So

$$
\left\|P\left(\sum_{i=1}^{n} x_{i} \otimes y_{i}\right)\right\|_{Y_{1} \otimes^{\wedge} Y_{2}} \leq\left\|P_{1}\right\|\left\|P_{2}\right\|\left\|\sum_{i=1}^{n} x_{i} \otimes y_{i}\right\|_{X_{1} \otimes^{\wedge} X_{2}} .
$$

And in the case of injective tensor product we have

$$
\begin{aligned}
\| P( & \left.\sum_{i=1}^{n} x_{i} \otimes y_{i}\right) \|_{Y_{1} \otimes \vee Y_{2}} \\
& =\|\left.\sum_{i=1}^{n} P_{1}\left(x_{i}\right) \otimes P_{2}\left(y_{i}\right)\right|_{Y_{1} \otimes \vee} Y_{2} \\
& =\sup \left\{\left|\sum_{i=1}^{n} f\left(P_{1}\left(x_{i}\right)\right) g\left(P_{2}\left(y_{i}\right)\right)\right|: f \in U_{Y_{1}^{*}}, g \in U_{Y_{2}^{*}}\right\} \\
& =\sup \left\{\left|f\left(P_{1}\left(\sum_{i=1}^{n} g\left(P_{2}\left(y_{i}\right)\right) x_{i}\right)\right)\right|: f \in U_{Y_{1}^{*}}, g \in U_{Y_{2}^{*}}\right\} \\
& \leq \sup \left\{\left\|P_{1}\right\|\left\|\sum_{i=1}^{n} g\left(P_{2}\left(y_{i}\right)\right) x_{i}\right\|_{X_{1}}: g \in U_{Y_{2}^{*}}\right\} \\
& =\left\|P_{1}\right\| \sup \left\{\sup \left\{\left|\sum_{i=1}^{n} f\left(x_{i}\right) g\left(P_{2}\left(y_{i}\right)\right)\right|: f \in U_{X_{1}^{*}}\right\}, g \in U_{Y_{2}^{*}}\right\} \\
& \leq\left\|P_{1}\right\|\left\|P_{2}\right\| \sup \left\{\left|\sum_{i=1}^{n} f\left(x_{i}\right) g\left(y_{i}\right)\right|: f \in U_{X_{1}^{*}}, g \in U_{X_{2}^{*}}\right\} \\
& \leq\left\|P_{1}\right\|\left\|P_{2}\right\|\left\|\sum_{i=1}^{n} x_{i} \otimes y_{i}\right\| X_{1} \otimes \vee X_{2}
\end{aligned}
$$

Thus in both cases, $\|P\| \leq\left\|P_{1}\right\|\left\|P_{2}\right\|$. Taking the infimum of each side with respect to all such $P_{1}$ and $P_{2}$, we get inequality (2.4). To prove inequality (2.5), we apply inequality (2.4) and get in particular

$$
\begin{aligned}
\lambda\left(X \otimes^{\vee} Y, l_{\infty}\left(U_{X^{*}}\right) \otimes^{\vee} l_{\infty}\left(U_{Y^{*}}\right)\right) & \leq \lambda\left(X, l_{\infty}\left(U_{X^{*}}\right)\right) \lambda\left(Y, l_{\infty}\left(U_{Y^{*}}\right)\right) \\
& =\lambda(X) \lambda(Y) .
\end{aligned}
$$

Using Lemma 2.2 and definition (1.2), we get $\lambda\left(X \otimes^{\vee} Y, l_{\infty}(\Omega)\right) \geq \lambda\left(X \otimes^{\vee} Y\right.$, $\left.l_{\infty}\left(U_{X^{*}}\right) \otimes^{\vee} l_{\infty}\left(U_{Y^{*}}\right)\right)$. We claim that the sign $\geq$ is an equal sign. In fact, if $P$ is any projection from $l_{\infty}\left(U_{X^{*}}\right) \otimes^{\vee} l_{\infty}\left(U_{Y^{*}}\right)$ onto $X \otimes^{\vee} Y$ and $J$ is the embedding given in Lemma 2.1 , then $P=P J$ is a projection from $l_{\infty}(\Omega)$ onto $X \otimes^{\vee} Y$ with $\|P\| \leq\|P\|$. This is the sufficient condition for the two infimum 
304 On the projection constants of some topological spaces

$\lambda\left(X \otimes^{\vee} Y, l_{\infty}(\Omega)\right)$ and $\lambda\left(X \otimes^{\vee} Y, l_{\infty}\left(U_{X^{*}}\right) \otimes^{\vee} l_{\infty}\left(U_{Y^{*}}\right)\right)$ to be equal. Therefore

$$
\lambda\left(X \otimes^{\vee} Y\right)=\lambda\left(X \otimes^{\vee} Y, l_{\infty}\left(U_{X^{*}} \otimes^{\vee} U_{Y^{*}}\right)\right)
$$

Using inequality (2.10), we get (2.5).

Remark 2.4. Since $\lambda\left(l_{\infty}(\Gamma)\right)=1$ for any index set $\Gamma$, we conclude that $\lambda\left(l_{\infty}(\Gamma)\right.$ $\left.\otimes^{(\vee \text { or } \wedge)} l_{\infty}(\Lambda), X \otimes^{(\vee \text { or } \wedge)} Y\right)=1$ for every $X \supset l_{\infty}(\Gamma)$ and $Y \supset l_{\infty}(\Lambda)$.

We have the following two corollaries.

Corollary 2.5. For any finite sequence $\left\{X_{i}\right\}_{i=1}^{n}$ of Banach spaces with complemented subspaces $\left\{Y_{i}\right\}_{i=1}^{n}$, the relative projection constant of the injective (resp., projective) tensor product $\bigotimes_{i=1}^{n} Y_{i}$ of the spaces $Y_{i}$ in the space $\bigotimes_{i=1}^{n} X_{i}$ satisfies

$$
\lambda\left(\bigotimes_{i=1}^{n} Y_{i}, \bigotimes_{i=1}^{n} X_{i}\right) \leq \prod_{i=1}^{n} \lambda\left(Y_{i}, X_{i}\right)
$$

Corollary 2.6. Let $\left\{Y_{i}\right\}_{i=1}^{n}$ be a finite sequence of finite-dimensional Banach spaces. Then the relation between the absolute projection constant of the projective (or injective) tensor product $\bigotimes_{i=1}^{n} Y_{i}$ and the direct sum $\sum_{i=1}^{n} \bigoplus Y_{i}$ (with the supremum norm) is as follows:

$$
\lambda\left(\bigotimes_{i=1}^{n} Y_{i}\right) \leq\left(\lambda\left(\sum_{i=1}^{n} \bigoplus Y_{i}\right)\right)^{n} .
$$

Proof. In fact, the proof is a combination of Corollary 2.5 and the results of [3, Theorem 4].

\section{Applications}

In this section, using Theorem 2.3, we obtain new results.

(1) For finite-dimensional Banach spaces $X$ and $Y$ with dimensions $n$ and $m$, respectively, we have

$$
\begin{aligned}
\lambda(X \otimes Y) \leq & \sqrt{n m}-\frac{1}{\sqrt{n m}}+O\left(n m^{-3 / 4}\right) \\
- & \left\{\left(\sqrt{m}-\frac{1}{\sqrt{m}}\right)\left(\frac{1}{\sqrt{n}}-O\left(n^{-3 / 4}\right)\right)\right. \\
& \left.+\left(\sqrt{n}-\frac{1}{\sqrt{n}}\right)\left(\frac{1}{\sqrt{m}}-O\left(m^{-3 / 4}\right)\right)\right\},
\end{aligned}
$$


in the real field and

$$
\begin{aligned}
\lambda(X \otimes Y) \leq & \sqrt{n m}-\frac{1}{2 \sqrt{n m}}+O\left(n m^{-3 / 4}\right) \\
- & \left\{\left(\sqrt{m}-\frac{1}{2 \sqrt{m}}\right)\left(\frac{1}{2 \sqrt{n}}-O\left(n^{-3 / 4}\right)\right)\right. \\
& \left.+\left(\sqrt{n}-\frac{1}{2 \sqrt{n}}\right)\left(\frac{1}{2 \sqrt{m}}-O\left(m^{-3 / 4}\right)\right)\right\},
\end{aligned}
$$

in the complex field. Compare this result with the result in (1.6).

(2) For any positive integer $m$ (not necessarily prime) with a prime factorization $m=\prod_{i=1}^{n} q_{i}$ where the numbers $q_{i}$ are distinct prime numbers, the space $\bigotimes_{i=1}^{n} l_{q_{i}^{2}}^{\infty}$ contains a subspace $Y$ of dimension $m$ with

$$
\lambda(Y) \leq \sqrt{\prod_{i=1}^{n} q_{i}}-\left(\frac{1}{\sqrt{\prod_{i=1}^{n} q_{i}}}-\frac{1}{\prod_{i=1}^{n} q_{i}}\right)-C(m),
$$

where $C(m)$ is a positive number depending on $m$ (in case of $m=q_{1} q_{2}, C(m)=$ $\left.\left[\left(1 / \sqrt{q_{1}}-1 / q_{1}\right)\left(\sqrt{q_{2}}-1 / \sqrt{q_{2}}\right)+\left(1 / \sqrt{q_{2}}-1 / q_{2}\right)\left(\sqrt{q_{1}}-1 / \sqrt{q_{1}}\right)\right]\right)$. Comparing this result with (1.3), we mention that the $m^{2}$-dimension of the space $\bigotimes_{i=1}^{n} l_{q_{i}^{2}}^{\infty}$ is not a square of a prime number, so it gives a new subspace $Y$ with a new projection constant.

(3) For numbers $p, q$ with $1 \leq p, q \leq 2$, we have

$$
\lim _{n, m \rightarrow \infty} \frac{\lambda\left(l_{p}^{n} \otimes l_{q}^{m}\right)}{\sqrt{n m}} \leq \begin{cases}\frac{2}{\pi}, & \text { in the real field, } \\ \frac{\pi}{4}, & \text { in the complex field }\end{cases}
$$

\section{The projection constants of operators}

Now we start with our basic definitions of the projection constants of operators.

Definition 4.1. (1) A linear bounded operator $A$ from a Banach space $X$ into a Banach space $Y$ is said to be left complemented with respect to a Banach space $Z$ ( $Z$ contains $Y$ as a closed subspace) if and only if there exists a linear bounded operator $B$ from $Z$ into $X$ such that the composition $A B$ is a projection from $Z$ onto $Y$. In this case $Z$ is said to be a left complementation of $A$.

If $P_{Z}(A)$ denotes the convex set of all operators $B$ from $Z$ into $X$ such that the composition $A B$ is a projection, then

(2) the left relative projection constant of the operator $A$ with respect to the space $Z$ is defined as

$$
\lambda_{l}(A, Z):=\inf \left\{\|A B\|: B \in P_{Z}(A)\right\} .
$$


(3) And the left absolute projection constant of $A$ is defined as $\lambda_{l}(A):=\sup \left\{\lambda_{l}(A, Z): Z\right.$ is a left complementation of the operator $\left.A\right\}$.

We define the same analogy from the right.

Remark 4.2. We notice the following.

(1) From the definition of $\lambda_{l}(A, Z)$, the infimum in (4.1) is taken only with respect to the projections that are factored (through $X$ ) into two operators one of them is $A$ and the other is an operator from $Z$ into $X$, so $1 \leq \lambda(Y, Z) \leq \lambda_{l}(A, Z)$ for every left complementation $Z$ of $A$.

(2) If $A$ is a projection from $X$ onto $Y$, then $A$ is left complemented with respect to $Y$. In fact $A J$ is a projection for any embedding $J$ from $Y$ into $X$.

(3) If $I_{Y}$ is the identity operator on $Y$ and $X$ contains $Y$ as a complemented subspace, then $I_{Y} P=P$ for every projection $P$ from $X$ onto $Y$ and hence $I_{Y}$ is left complemented with respect to $X$. Moreover, $\lambda_{l}\left(I_{Y}, X\right)=\lambda(Y, X)$, that is, the relative projection constant of the identity operator on the space $Y$ with respect to the space $X$ is the relative projection constant of the space $Y$ in the space $X$.

(4) If $Z$ is a left complementation of the linear bounded operator $A: X \rightarrow Y$, then $Y$ is complemented in $Z$ and the operator $A$ is onto.

(5) If $Z$ is a separable or reflexive Banach space and $X$ is a Banach space, then for any index set $\Gamma$ the space $Z$ is not a right complementation of any linear bounded operator from $l_{\infty}(\Gamma)$ into $X$. In particular, if $X$ is a Banach space, then for any index set $\Gamma$, the space $l_{\infty}(\Gamma)$ is not a left complementation of any linear bounded operator from $X$ into the space $c_{0}$.

The following lemma is parallel to that lemma mentioned in [8] for Banach spaces and we omit the proof since the proof is nearly similar.

LEMMA 4.3. Let $\Gamma$ be an index set such that $Y$ is isometrically embedded into $l_{\infty}(\Gamma)$ and let $A$ be a linear bounded operator from $X$ onto $Y$ such that $l_{\infty}(\Gamma)$ is one of its left complementation. Then for a given $B \in P_{l_{\infty}(\Gamma)}(A)$,

(1) For all Banach spaces $E, Z, E \subseteq Z$ and every linear bounded operator $T$ from $E$ into $Y$ there is an operator $\hat{T}$ from $Z$ into $Y$ extending the operator $T$ with $\|\hat{T}\| \leq\|A B\|\|T\|$, that is, the space $Y$ has $\|A B\|$-extension property, and in particular, if $Z \supseteq X$, the operator $A$ has a linear extension $\hat{A}$ from $Z$ into $Y$ with $\|\hat{A}\| \leq\|A B\|\|A\|$. That is, the extension constant $c(A)$ of the operator $A$ defined by $\left(c(A):=\sup _{X \subset Z} \inf \{\|\hat{A}\|: \hat{A}\right.$ is an extension of $A$ and $\left.\hat{A}: Z \rightarrow Y\}\right)$ satisfies $c(A) \leq\|A B\|\|A\|$.

(2) For every Banach space $Z \supseteq Y$, there exists a projection $P$ from $Z$ onto $Y$ such that $\|P\| \leq\|A B\|$.

The following theorem is also parallel to that given in (1.3) for Banach spaces. 
THEOREM 4.4. Let $Y$ be isometrically embedded in $l_{\infty}(\Gamma)$ and let $A$ be a linear bounded operator from $X$ onto $Y$ such that $l_{\infty}(\Gamma)$ is a left complementation of A. Then A is left complemented with respect to any other Banach space $Z$ containing $Y$ as a closed subspace. Moreover,

$$
\lambda_{l}(A, Z) \leq \lambda_{l}\left(A, l_{\infty}(\Gamma)\right)
$$

for every Banach space $Z$ containing $Y$ as a closed subspace, that is, $\lambda_{l}(A)$ attains its supremum at $l_{\infty}(\Gamma)$. Therefore,

$$
\lambda_{l}(A)=\lambda_{l}\left(A, l_{\infty}(\Gamma)\right), \quad c(A) \leq\|A\| \lambda_{l}(A) .
$$

\section{References}

[1] M. M. Day, Normed Linear Spaces, Springer-Verlag, Berlin, 1958. MR $20 \# 1187$. Zbl 082.10603.

[2] D. J. H. Garling and Y. Gordon, Relations between some constants associated with finite dimensional Banach spaces, Israel J. Math. 9(1971), 346-361. MR 54\#896. Zbl 212.14203.

[3] Y. Gordon, On the projection and Macphail constants of $l_{n}{ }^{p}$ spaces, Israel J. Math. 6 (1968), 295-302. MR 38\#4961. Zbl 182.45202.

[4] B. Grünbaum, Projection constants, Trans. Amer. Math. Soc. 95 (1960), 451-465. MR 22\#4937. Zbl 095.09002.

[5] H. Jarchow, Locally Convex Spaces, Mathematical Textbooks, Teubner, Stuttgart, 1981. MR 83h:46008. Zbl 466.46001.

[6] M. I. Kadets and M. G. Snobar, ber gewisse Funktionale auf einem Minkowskischen Kompaktum [Certain functionals on the Minkowski compactum], Mat. Zametki 10 (1971), 453-457 (Russian), translated in Math. Notes 10 (1971) 694-696. MR 45\#861. Zbl 229.46018.

[7] H. König, Spaces with large projection constants, Israel J. Math. 50 (1985), no. 3, 181-188. MR 86g:46023. Zbl 582.46012.

[8] _ Eigenvalue Distribution of Compact Operators, Operator Theory: Advances and Applications, vol. 16, Birkhäuser Verlag, Stuttgart, 1986. MR 88j:47021. Zbl 618.47013.

[9] H. König and D. R. Lewis, A strict inequality for projection constants, J. Funct. Anal. 74 (1987), no. 2, 328-332. MR 88j:46014. Zbl 631.46023.

[10] H. König, C. Schütt, and N. Tomczak-Jaegermann, Projection constants of symmetric spaces and variants of Khintchine's inequality, J. Reine Angew. Math. 511 (1999), 1-42. MR 2000i:46014. Zbl 926.46008.

[11] H. König and N. Tomczak-Jaegermann, Bounds for projection constants and 1-summing norms, Trans. Amer. Math. Soc. 320 (1990), no. 2, 799-823. MR 90k:46028. Zbl 713.46012.

[12] _ Norms of minimal projections, J. Funct. Anal. 119 (1994), no. 2, 253-280. MR 94m:46024. Zbl 818.46015.

[13] D. R. Lewis, Finite dimensional subspaces of $L_{p}$, Studia Math. 63 (1978), no. 2, 207-212. MR 80b:46043. Zbl 406.46023.

[14] , An upper bound for the projection constant, Proc. Amer. Math. Soc. 103 (1988), no. 4, 1157-1160. MR 89g:46042. Zbl 659.46010. 
308 On the projection constants of some topological spaces

[15] D. Rutovitz, Some parameters associated with finite-dimensional Banach spaces, J. London Math. Soc. 40 (1965), 241-255. MR 32\#8120. Zbl 125.06402.

[16] H. H. Schaefer, Topological Vector Spaces, Maxmillan Series in Advanced Mathematics and Theoretical Physics, The Macmillan, New York, 1966. MR 33\#1689. Zbl 141.30503.

Entisarat El-Shobaky: Department of Mathematics, Faculty of Science, Ain Shams University, Cairo, Egypt

E-mail address: solar@ photoenergy.org

Sahar Mohammed Ali: Department of Mathematical and Computing Sciences, Tokyo Institute of Technology, 2-12-1 Ookayama, Meguro-Ku, Tokyo 152-8552, JAPAN

E-mail address: sahar0@is.titech.ac.jp

Wataru Takahashi: Department of Mathematical and Computing Sciences, Tokyo Institute of Technology, 2-12-1 Ookayama, Meguro-Ku, Tokyo 152-8552, JAPAN

E-mail address: wataru@is.titech.ac.jp 


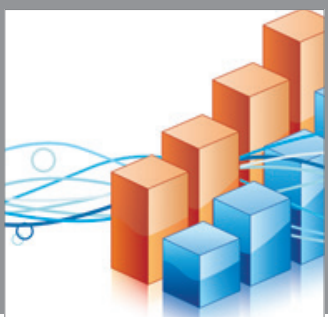

Advances in

Operations Research

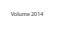

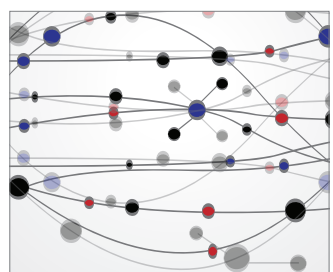

\section{The Scientific} World Journal
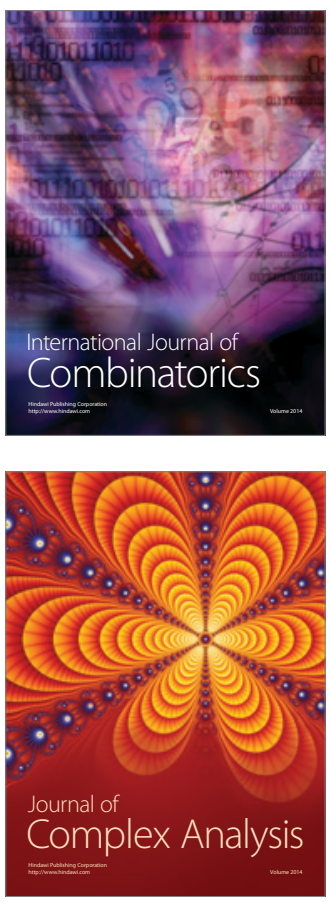

International Journal of

Mathematics and

Mathematical

Sciences
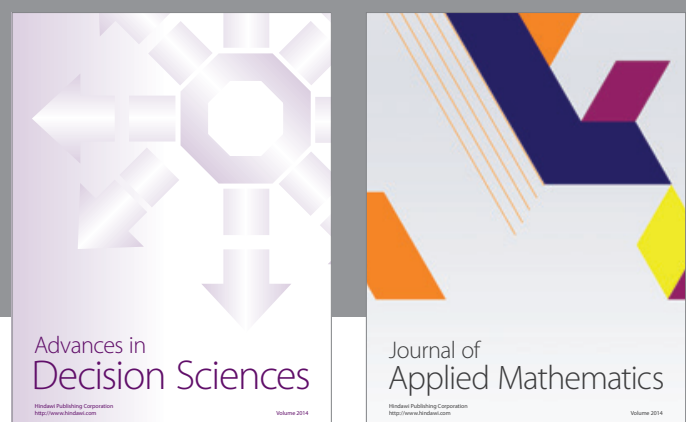

Journal of

Applied Mathematics
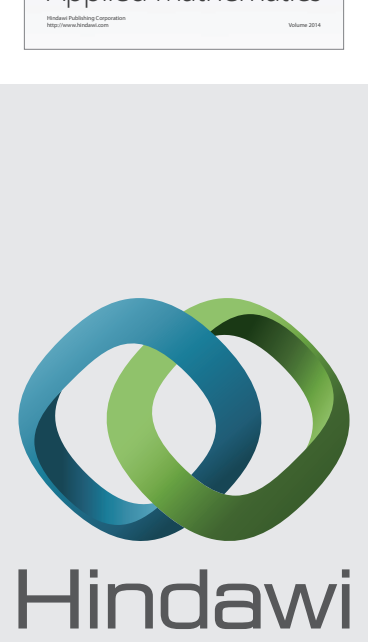

Submit your manuscripts at http://www.hindawi.com
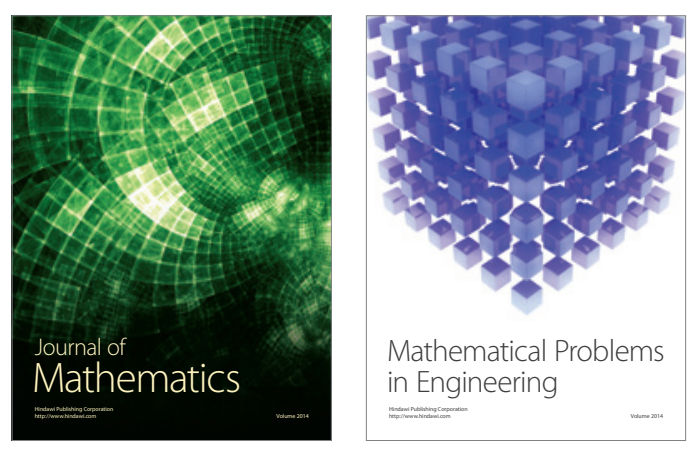

Mathematical Problems in Engineering
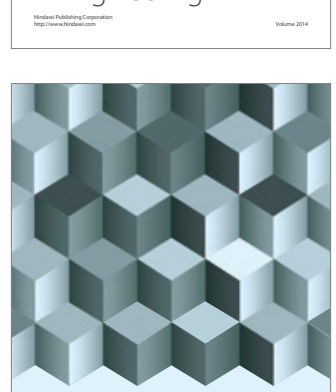

Journal of

Function Spaces
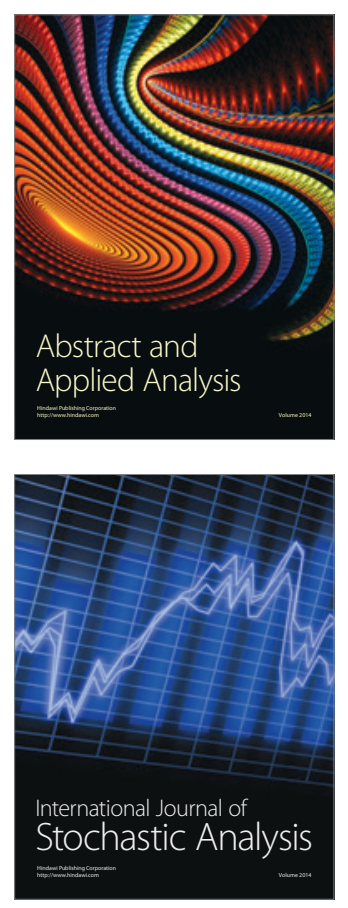

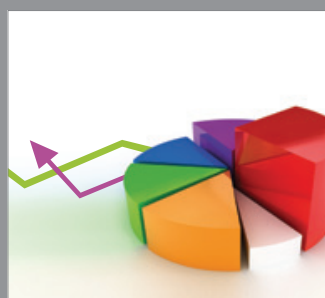

ournal of

Probability and Statistics

Promensencen
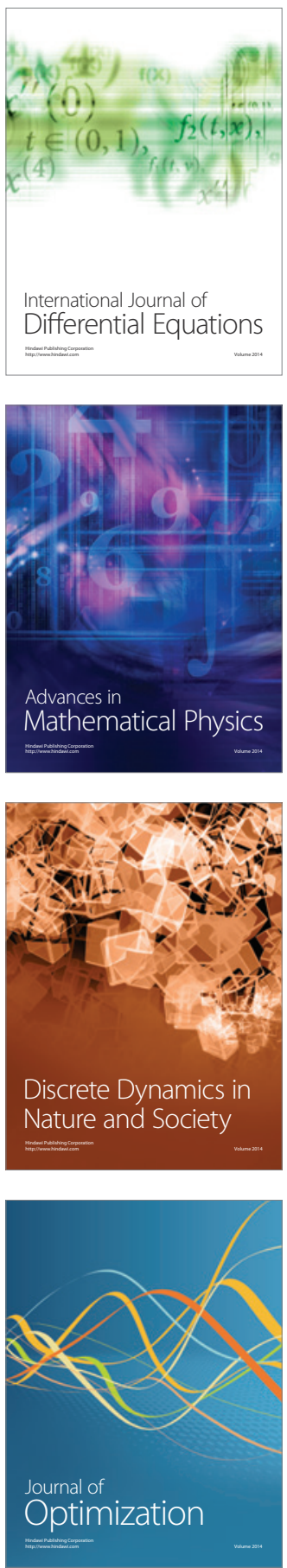